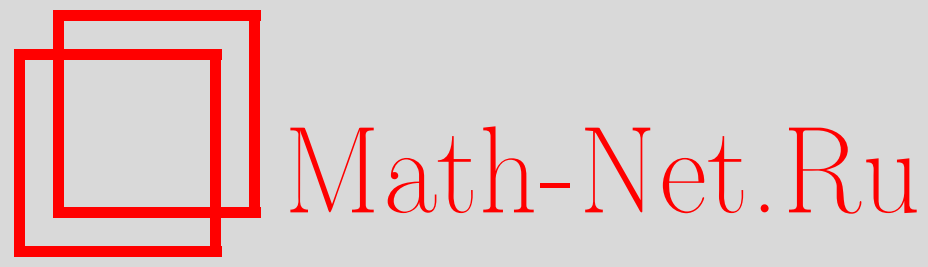

Б. С. Дарховский, М. Старосвицкий, О задаче различения двух гипотез при наличии неизвестного параметра, Теория вероятн. и ее примен., 2002, том 47, выпуск 4, 654-671

DOI: https://doi.org/10.4213/tvp3773

Использование Общероссийского математического портала MathNet.Ru подразумевает, что вы прочитали и согласны с пользовательским соглашением

http://www . mathnet.ru/rus/agreement

Параметры загрузки:

IP : 35.173 .219 .149

26 апреля 2023 г., 14:30:19

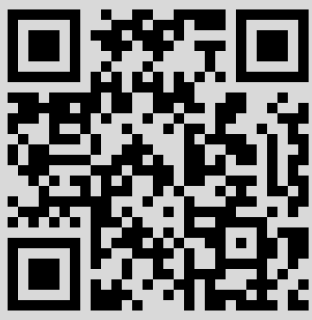


(c) 2002 г. ДАРХОВСКИЙ Б. С.*, СТАРОСВИЦКИЙ М. ${ }^{* *}$

\section{О ЗАДАЧЕ РАЗЛИЧЕНИЯ ДВУХ ГИПОТЕЗ ПРИ НАЛИЧИИ НЕИЗВЕСТНОГО ПАРАМЕТРА ${ }^{1)}$}

Рассматривается задача проверки гипотез о принадлежности выборки к одному из двух семейств распределений. Каждое семейство задано плотностью, зависяшей от конечномерного параметра, пробегающего некоторое заданное компактное множество. Предлагаются два решаюших правила: первое основано на идее использования части выборки для принятия решения и всей выборки для оценивания параметра, а второе, кроме идеи принятия решения по части выборки, использует усреднение по искусственно построенному «апостериорному» распределению параметра вместо его оценивания. Доказано, что предлагаемые методы асимптотически эквивалентны оптимальному байесовскому правилу для случая, когда параметр известен. Рассмотрена также задача о различении гипотез, когда параметр, задаюший плотность, принимает значения из двух заданных множеств.

Ключевье слова и фразы: проверка гипотез, параметрическое семейство плотностей, апостериорное распределение.

1. Введение. Пусть $\mathbf{P}_{i}, i=0,1$, - две вероятностные меры, имеющие плотности $f_{i}(x, \theta)$ соответственно по отношению к некоторой $\sigma$-конечной мере $\mu$ и зависящие от неизвестного конечномерного параметра $\theta$.

Рассмотрим наборы вероятностных распределений, порождаемые плотностями $f_{i}$, когда параметр $\theta$ пробегает некоторое заданное множество $\Theta$, содержащееся в некотором открытом подмножестве $U$ конечномерного пространства. Иными словами, $\mathscr{P}_{i}=\left\{f_{i}(x, \theta)\right\}, \theta \in \Theta, i=0,1$.

Мы будем интересоваться задачей различения гипотез о принадлежности простой выборки (т.е. выборки, состоящей из независимых и одинаково распределенных случайных величин) $X=\left(x_{1}, \ldots, x_{n}\right)$ к семейству $\mathscr{P}_{0}\left(\right.$ гипотеза $\left.\mathbf{H}_{0}\right)$ или $\mathscr{P}_{1}\left(\right.$ гипотеза $\left.\mathbf{H}_{1}\right)$.

*Институт системного анализа РАН, пр-т 60-летия Октября, 9, 117312 Москва, B-312, Россия; e-mail: darbor@isa.ru

**LAIL CNRS UPRESA 8021 EUDIL Université des Sciences et Technologies de Lille 59655 Villeneuve d'Ascq cedex, France; e-mail: staroswiecki@univ-lille1.fr

1) Работа выполнена при поддержке гранта (PAST RI) Министерства исследований Франции. 
Мы рассмотрим также аналогичную задачу различения гипотез для случая, когда заданы два (непересекающихся) параметрических множества $\Theta_{0}, \Theta_{1}$ и одно параметрическое семейство плотностей $\{f(x, \theta)\}$, $\theta \in \Theta=\Theta_{0} \cup \Theta_{1}$. В обоих вариантах предполагается, что параметрические семейства плотностей $f_{i}, i=0,1$, и $f$ известны и определены для всех значений параметра из множества $U$. Всюду далее термин «параметрическое пространство» заменяет термин «открытое подмножество $U$ конечномерного пространства».

Наш интерес к проблемам такого рода был вызван прикладными задачами обнаружения изменений в динамических системах (в англоязычной литературе такие задачи называют «fault detection-isolation problems» или «FDI»). Дело в том, что вектор состояния динамической системы обычно наблюдается не полностью и со случайными помехами, так что распределения наблюдаемых данных зависят от вектора состояния как от параметра. Изменения в динамической системе могут быть вызваны изменением распределений случайных ошибок, появлением новых внешних воздействий, изменениями параметров системы или совокупностью этих причин. Таким образом, наблюдения обычно имеют вид

$$
x_{n}=\varphi\left(\theta, \xi_{n}\right)
$$

где $\xi_{n}$ - независимые случайные величины, плотность распределения которых определяется состоянием динамической системы, а для вектора состояния $\theta$ при естественных предположениях можно заранее дать описание множества, к которому он принадлежит (или множеств, в случае, когда происходят изменения системных параметров или возникновение новых внешних воздействий). Тогда FDI-проблема может быть сведена к задаче различения статистических гипотез (нулевая гипотеза - изменений нет, «номинальный режим», альтернатива - произошли какиелибо из отмеченных выше изменений, режим сбоев) именно такого типа, о котором говорилось выше, т.е. при наличии неизвестного параметра, который может принадлежать одному или двум (в простейшем случае) заранее заданным множествам.

В прикладной литературе для решения таких задач используется эвристический прием, состоящий в том, что (при различении двух гипотез) в критерий отношения правдоподобия вместо параметра подставляется соответствующая оценка максимального правдоподобия (ОМП). Однако никаких утверждений об оптимальности такого рода критериев не приводится.

В математической статистике задачи проверки сложных параметрических гипотез также привлекают большое внимание. Обычно рассматривается задача проверки гипотез с двумя параметрическими множествами. По-видимому, первые результаты об асимптотической оптимальности критерия отношения правдоподобия, в который вместо па- 
раметра подставлена его ОМП, построенная по всей выборке, для проверки гипотез при наличии двух параметрических множеств содержатся в работе [1]. В этой работе показано, что на критерии отношения правдоподобия достигается нижняя асимптотическая граница вероятности ошибки первого рода. В [3, с. 349-350] отмечается, что «точных свойств оптимальности этот критерий отношения правдоподобия в общем случае, по-видимому, не имеет». Там же, на с. 373-407, указаны случаи, когда удается устанавливать асимптотическую оптимальность такого критерия при специальных условиях (например, в предположении о сближающихся гипотезах). В работе [2] для задачи с двумя параметрическими множествами предложено довольно сложное преобразование параметрического пространства, которое, при определенных условиях, позволяет построить в новом параметрическом пространстве тест отношения правдоподобия, асимптотически оптимальный в отношении обеих вероятностей ошибок.

Насколько нам известно, задачи с одним параметрическим множеством в литературе по математической статистике не рассматривались.

В этой статье мы предлагаем две новые идеи для задач указанного класса. Первая идея состоит в том, чтобы решение о гипотезе принималось на основе только части наблюдений $\tilde{n}<n$, а оценка параметра производилась по всей выборке. На первый взгляд, такой способ кажется странным. Однако ему можно дать разумную трактовку. А именно, можно с самого начала считать, что мы имеем дело с выборкой объема $\tilde{n}<n$, а «дополнительные» $n-\tilde{n}$ наблюдений требуются для того, чтобы критерий отношения правдоподобия после подстановки в него соответствующей оценки параметра стал асимптотически эквивалентным оптимальному правилу, построенному для случая, когда параметр точно известен. Иными словами, можно полагать, что «дополнительные» наблюдения есть та «плата», которую необходимо внести для достижения оптимальности при проверке гипотез с выборкой объема $\widetilde{n}$. Другая (и, возможно, наиболее интересная) трактовка такова. Если рассматривать описанные выше задачи в последовательном варианте (т.е. как задачи о «разладке»), то целесообразно использовать два «движущихся окна» наблюдений: «большое» (для оценки параметра) и «маленькое» (для принятия решения) с тем, чтобы уменьшить запаздывание в обнаружении разладки при сохранении приемлемого уровня «ложных тревог».

Оказывается, для достаточно широкого класса ситуаций при естественных условиях и любом выборе $\tilde{n}<n, \tilde{n} / n \rightarrow 0$ при $n \rightarrow \infty, \tilde{n} \rightarrow \infty$ (например, при $\tilde{n}=n^{\varepsilon}, 0<\varepsilon<1$ ) указанная асимптотическая эквивалентность имеет место; при этом не требуются дополнительные предположения, например, о сближении гипотез. Хотя такое правило теоретически проигрывает оптимальному (которое для задач с одним параметрическим множеством еще неизвестно!), нам представляется, что 
оно может оказаться практически полезным, так как реальная эффективность оптимального правила определяется скоростью сходимости в асимптотических соотношениях, и эта реальная эффективность может оказаться сопоставимой с той, которая получается при использовании предлагаемого правила, поскольку отношение части выборки, используемой для принятия решения, ко всей выборке, сколь угодно медленно стремится к нулю.

Вторая идея, которая предлагается в статье, состоит в том, чтобы использовать некоторый «усредненный» критерий отношения правдоподобия, причем усреднение проводится по искусственно построенной на параметрическом множестве «апостериорной мере» (хотя сам параметр не предполагается случайным, такую меру можно построить, и она будет обладать всеми свойствами апостериорного распределения). Отметим, что в «усредненный» критерий отношения правдоподобия снова подставляется часть выљорки, т.е. вновь используется и первая идея. И снова (при том же соотношении между $n$ и $\tilde{n}$ ) удается установить асимптотическую эквивалентность такого метода оптимальному, построенному для случая, когда параметр точно известен. Более того, при помощи этой идеи можно предложить новый метод и установить такую же асимптотическую эквивалентность его оптимальному методу для задачи с двумя параметрическими множествами.

Организация статьи такова. В п. 2 мы рассматриваем задачу с одним параметрическим множеством и критерий, основанный на первой идее. В п. 3 такая же задача решается с использованием второй идеи. В п. 4 приводится результат о задаче с двумя параметрическими множествами и дается краткое обсуждение предложенных методов.

2. Первое решающее правило. В этом и следующем пунктах мы будем рассматривать задачу проверки гипотез с одним параметрическим множеством. Для корректной постановки задачи необходимо предположить, что выполняется условие детектируемости

$$
\mathscr{P}_{0} \cap \mathscr{P}_{1}=\varnothing \text {. }
$$

Ниже мы приведем достаточное условие для (1).

Всюду далее в работе мы будем использовать так называемые условия регулярности для плотностей (см. [3, с. 238-239]). В целях экономии места мы не приводим точной формулировки этих условий, отсылая читателя к [3], и напомним только, что, грубо говоря, условия регулярности означают достаточную гладкость плотностей по параметру и существование соответствующих матриц Фишера.

Обозначим через $\mathbf{P}_{\theta^{\star}, 1}\left(\mathbf{E}_{\theta^{\star}, 1}\right), \mathbf{P}_{\theta^{\star}, 0}\left(\mathbf{E}_{\theta^{\star}, 0}\right)$ вероятностные меры (математические ожидания), отвечающие соответственно гипотезам $\mathbf{H}_{1}, \mathbf{H}_{0}$ и «истинному» значению параметра $\theta^{\star}$. 
Выберем в параметрическом пространстве шар $K$, содержащий множество $\Theta$, и скажем, что $\hat{\theta}_{1}, \hat{\theta}_{0}$ есть специальные оченки максимального правдоподобия (СОМП) для неизвестного параметра $\theta$, если они являются проекциями на компакт $K$ обычных ОМП, вычисленных $n o$ всей выборке $X$ и без ограничений на параметр в соответствии с гипотезами $\mathbf{H}_{1}, \mathbf{H}_{0}$. Таким образом, по определению, СОМП не покидают компакт $K$, но не обязаны принадлежать множеству $\Theta$.

Рассмотрим следующее условие: в параметрическом пространстве существуют множества $Z_{0} \in K$ и $Z_{1} \in K$ такие, что

$$
\inf _{z \in Z_{0}} \operatorname{dis}(z, \Theta) \geqslant \delta>0, \quad \inf _{z \in Z_{1}} \operatorname{dis}(z, \Theta) \geqslant \delta>0
$$

и при любом $x>0$

$$
\begin{aligned}
& \lim _{n \rightarrow \infty} \mathbf{P}_{\theta^{\star}, 1}\left\{\operatorname{dis}\left(\hat{\theta}_{0}, Z_{0}\right)>x\right\}=0, \\
& \lim _{n \rightarrow \infty} \mathbf{P}_{\theta^{\star}, 0}\left\{\operatorname{dis}\left(\hat{\theta}_{1}, Z_{1}\right)>x\right\}=0
\end{aligned}
$$

для любого $\theta^{\star} \in \Theta$, где $\operatorname{dis}(u, \Theta) \triangleq \inf _{\theta \in \Theta}\|u-\theta\|$.

Легко видеть, что из (2) следует (1), если условия регулярности выполнены для некоторой окрестности множества $\Theta$. Действительно, пусть (2) верно, но (1) не выполняется. Это означает, что сушествуют точки $\theta^{\prime} \in \Theta, \theta^{\prime \prime} \in \Theta$ такие, что $f_{1}\left(\cdot, \theta^{\prime}\right)=f_{0}\left(\cdot, \theta^{\prime \prime}\right) \mu$-п.н. Поэтому $\mathbf{P}_{\theta^{\prime}, 1} \equiv \mathbf{P}_{\theta^{\prime \prime}, 0}$. Но тогда

$$
0=\lim _{n \rightarrow \infty} \mathbf{P}_{\theta^{\prime}, 1}\left\{\operatorname{dis}\left(\hat{\theta}_{0}, Z_{0}\right)>\frac{\delta}{2}\right\}=\lim _{n \rightarrow \infty} \mathbf{P}_{\theta^{\prime \prime}, 0}\left\{\operatorname{dis}\left(\hat{\theta}_{0}, Z_{0}\right)>\frac{\delta}{2}\right\}=1,
$$

так как $\hat{\theta}_{0} \stackrel{\mathbf{P}_{\theta^{\prime \prime}, 0}}{\longrightarrow} \theta^{\prime \prime} \in \Theta, \operatorname{dis}\left(\theta^{\prime \prime}, Z_{0}\right)>\delta$ в силу условий регулярности. Аналогичным образом рассматривается и оценка $\hat{\theta}_{1}$. Мы получили противоречие с (2).

Условие (2) заслуживает более подробного обсуждения, которое, без ограничения общности, удобно провести на примере. Допустим, что имеется тройка величин $\left(\theta_{1}, \theta_{2}, \theta_{3}\right)$ таких, что для плотности $f_{0}$ параметром является пара $\left(\theta_{1}, \theta_{2}\right)$, а $\theta_{3}$ известно, а для плотности $f_{1}$ - параметром является пара $\left(\theta_{2}, \theta_{3}\right)$, а $\theta_{1}$ известно, и обе эти пары принимают значения из одного и того же множества $\Theta$ (в пространстве соответствующей размерности; ясно, что размерности $\theta_{1}$ и $\theta_{3}$ совпадают). Тогда условие (2) будет выполнено, если:

a) значение $\theta_{3}$ для плотности $f_{0}$ (скажем, $a$ ) не принадлежит множеству значений параметра $\theta_{1}$ для той же плотности, а значение $\theta_{1}$ (скажем, $b$ ) для плотности $f_{1}$ не принадлежит множеству значений параметра $\theta_{3}$ (в общем для переменных $\theta_{1}$ и $\theta_{3}$ пространстве); в этом случае в качестве множества $Z_{1}$ можно взять множество $\left\{\theta_{1}=a\right\}$ в пространстве пар $\left(\theta_{1}, \theta_{2}\right)$, а в качестве множества $Z_{0}$ можно взять множество $\left\{\theta_{3}=b\right\}$ в пространстве пар $\left(\theta_{2}, \theta_{3}\right)$; 
б) статистика, используемая для построения ОМП-оценки параметра $\theta_{3}$ при гипіотезе $\mathbf{H}_{1}$, является состоятельной для этого же параметра при гипотезе $\mathbf{H}_{0}$, а статистика, используемая для построения ОМПоценки параметра $\theta_{1}$ при гипотезе $\mathbf{H}_{0}$, является состоятельной для этого же параметра при гипотезе $\mathbf{H}_{1}$.

В свою очередь, для выполнения б) достаточно, чтобы статистики, применяемые для оценивания параметра $\theta_{3}$ и параметра $\theta_{1}$, были (как функции наблюдений) одними и теми же как для $f_{0}$, так и для $f_{1}$. Но это всегда выполнено, если природа каждой из переменных $\theta_{1}$ и $\theta_{3}$ одна $u$ та же как для $f_{0}$, так и для $f_{1}$.

Заметим, что в описываемой ситуации можно с самого начала считать, что оценки $\hat{\theta}_{0}$ и $\hat{\theta}_{1}$ в условии $(2)$ - это просто какие-то асимптотически нормальные оценки, принимающие значения из шара $K$.

Таким образом, при наличии в наборе параметров таких элементов, которые для одной плотности играют роль неизвестного параметра, а для другой известны (и наоборот), причем природа таких элементов одна и та же для обеих плотностей, и при непересечении множеств значений этих элементов (что, по сути, необходимо для детектируемости) условие (2) заведомо гарантировано предположениями регулярности.

Рассмотрим теперь ситуацию, когда весь параметрический вектор $\theta \in \Theta$ имеет одну и ту же природу для обеих плотностей. Допустим сначала, что существуют гомеоморфизмы $\varphi_{i}: \Theta \rightarrow U, i=0,1$, такие, что плотности имеют вид $f_{0}\left(\varphi_{0}(\theta), x\right), f_{1}\left(\varphi_{1}(\theta), x\right)$. Пусть $\widehat{u}-$ некоторая состоятельная оценка нового параметра $u=\varphi_{i}(\theta)$ (одинакового для обеих плотностей) такая, что $\hat{\theta}_{i}=\varphi_{i}^{-1}(\widehat{u}), i=0,1$, - асимптотически нормальные оценки параметра $\theta$. Тогда

$$
\hat{\theta}_{0} \stackrel{\mathbf{P}_{\theta, 1}}{\longrightarrow}\left(\varphi_{0}^{-1} \circ \varphi_{1}\right)(\theta), \quad \hat{\theta}_{1} \stackrel{\mathbf{P}_{\theta, 0}}{\longrightarrow}\left(\varphi_{1}^{-1} \circ \varphi_{0}\right)(\theta) .
$$

Поэтому условие (2) будет выполнено, если

$$
\left(\varphi_{0}^{-1} \circ \varphi_{1}\right)(\Theta) \cap \Theta=\varnothing, \quad\left(\varphi_{1}^{-1} \circ \varphi_{0}\right)(\Theta) \cap \Theta=\varnothing
$$

(здесь $\left.Z_{0}=\left(\varphi_{0}^{-1} \circ \varphi_{1}\right)(\Theta), Z_{1}=\left(\varphi_{1}^{-1} \circ \varphi_{0}\right)(\Theta)\right)$.

$\mathrm{B}$ частности, если $\varphi_{i}(\theta)=u_{i}+\theta, i=0,1$, то условие (2) выполняется, если $\left(\Theta \pm\left(u_{1}-u_{0}\right)\right) \cap \Theta=\varnothing$.

Пусть теперь параметр $\theta$ имеет одну и ту же природу для обеих плотностей и условие (2) не выполнено, но найдутся хотя бы две такие характеристики $u \in \mathbf{R}^{k}, v \in \mathbf{R}^{k}$, которые различны для распределений, но природа которых одна и та же в каждой из плотностей. Тогда можно добиться его выполнения, расширив параметрическое пространство. А именно, пусть $u \in \mathbf{Q}, v=a \notin \mathbf{Q}$ для гипотезы $\mathbf{H}_{0}$ и $u=b \notin \mathbf{Q}$, $v \in \mathbf{Q}$ для гипотезы $\mathbf{H}_{1}$. Тогда можно рассмотреть тройку $(u, \theta, v)$ и объявить новым параметром для $f_{0}$ пару $(u, \theta)$, а для $f_{1}-$ пару $(\theta, v)$ 
(природа параметра $u$ и природа параметра $v$ для каждой из плотностей одна и та же). Тогда мы получаем рассмотренную выше схему с общим параметрическим множеством в расширенном пространстве и условие (2) выполняется. Заметим, что при этом параметры $u$ для $f_{0}$ и $v$ для $f_{1}$ могут быть известны, но все равно надо использовать их статистические оценки для того, чтобы решающее правило было асимптотически оптимальным.

Если описанный прием не проходит, а условие (2) не выполнено, то, скорее всего, информация о параметре не влияет на качество решающего правила и надо применять иные способы различения гипотез.

Таким образом, проведенный анализ позволяет сделать вывод, что в достаточно широком классе случаев предположение (2) выполняется.

Принимая во внимание эти замечания, мы будем предполагать всюду далее, не оговаривая этого специально, что для рассматриваемой задачи выполнены следующие условия:

1) множество $\Theta$ есть компакт в $\mathbf{R}^{k}$;

2) для всех $\theta \in \Theta_{r}$ и обеих плотностей выполнены условия регулярности (здесь $\Theta_{r}-r$-окрестность множества $\Theta$ для некоторого $r>0$ );

3) выполнено условие (2).

Кроме того, также специально не оговаривая, мы будем предполагать, что рассматриваемые далее интегралы (например, интегралы отношения правдоподобия по заданным плотностям и т.п.) существуют.

Мы формулируем задачу проверки гипотез в байесовской постановке, иными словами, если $d(X)$ - решающая функция (т.е. измеримое отображение выборочного пространства в множество $\{0,1\}), \alpha>0$, $\beta>0$ - заданные числа, то нас будет интересовать задача минимизации (при любом $\theta \in \Theta$ ) функционала:

$$
J=\alpha \mathbf{P}_{\theta, 1}\{d(X)=0\}+\beta \mathbf{P}_{\theta, 0}\{d(X)=1\} .
$$

Числа $\alpha, \beta$ в (3) можно трактовать как априорные вероятности гипотез (при условии, что $\alpha+\beta=1$ ), хотя такая трактовка не обязательна: эти числа можно рассматривать просто как весовые коэффициенты при соответствующих вероятностях ошибок.

Пусть $\tilde{n}$ таково, что $\tilde{n}<n$ и $\tilde{n} / n \rightarrow 0$ при $n \rightarrow \infty, \tilde{n} \rightarrow \infty$. Рассмотрим часть наблюдений $\tilde{X}=\left(x_{1}, \ldots, x_{\tilde{n}}\right)$.

$\mathrm{B}$ наших условиях

$$
f_{1}(\tilde{X}, \theta)=\prod_{i=1}^{\tilde{n}} f_{1}\left(x_{i}, \theta\right), \quad f_{0}(\tilde{X}, \theta)=\prod_{i=1}^{\tilde{n}} f_{0}\left(x_{i}, \theta\right) .
$$

Пусть $\theta^{*} \in \Theta$ - истинное (неизвестное) значение параметра. Положим

$$
D_{1}=\left\{\tilde{X}: \frac{\alpha f_{1}\left(\tilde{X}, \theta^{\star}\right)}{\beta f_{0}\left(\tilde{X}, \theta^{\star}\right)}>1\right\}, \quad D_{0}=\left\{\tilde{X}: \frac{\alpha f_{1}\left(\tilde{X}, \theta^{\star}\right)}{\beta f_{0}\left(\tilde{X}, \theta^{\star}\right)} \leqslant 1\right\} .
$$


Легко видеть, что если параметр $\theta^{\star}$ известен и только часть наблюдении $\widetilde{X}$ используется для принятия решения, то оптимальное байесовское правило заключается в выборе $\mathbf{H}_{1}$ на множестве $D_{1}$ и $\mathbf{H}_{0}$ на множестве $D_{0}$.

Теперь целесообразно изменить точку зрения на нашу задачу. А именно, допустим, что мы с самого начала имеем выборку объема $\widetilde{n}<n$ и хотим принять решение о гипотезах по этой выборке, но так, чтобы это решение было асимптотически эквивалентно оптимальному для любого (неизвестного!) $\theta \in \Theta$. Как уже отмечалось во введении, подстановка в критерий отношения правдоподобия ОМП параметра, построенных по той же выборке, по которой принимается решение о гипотезах, вообще говоря, не даст асимптотической оптимальности решающего правила. Но если использовать ОМП параметра, построенные по выборке несколько бо́льшего объема (т.е. по всем реально имеюшимся $n>\tilde{n}$ наблюдениям), то, возможно, такую оптимальность удастся установить. Далее мы займемся реализацией этой программы.

Рассмотрим следующую оценку:

$$
\tilde{\theta}=\frac{A_{1}}{A_{0}+A_{1}} \hat{\theta}_{0}+\frac{A_{0}}{A_{0}+A_{1}} \hat{\theta}_{1},
$$

где $A_{1}=\left(\operatorname{dis}\left(\hat{\theta}_{1}, \Theta\right)\right), A_{0}=\left(\operatorname{dis}\left(\hat{\theta}_{0}, \Theta\right)\right)$ (неопределенность вида $\frac{0}{0}$ в $(4)$ интерпретируется как $\left.\frac{1}{2}\right)$.

Всюду далее в статье мы будем использовать одну и ту же букву $C$ для обозначения, вообще говоря, разных положительных констант, не зависящих от $n$.

Лемма 1. При сделанньх предположениях существует положительная константа $C$ такая, что для любого $x>0$ и любого $\theta^{*} \in \Theta$

$$
\lim _{n \rightarrow \infty} \mathbf{P}_{\theta^{*}, i}\left\{\sqrt{n}\left\|\tilde{\theta}-\theta^{*}\right\|>x\right\} \leqslant C \exp \left(-C x^{2}\right), \quad i=0,1 .
$$

Д ок а за т ельст в о. Докажем соотношение (5) для $i=0$. Пусть $L$ - диаметр шара $K$. Тогда

$$
\left\|\tilde{\theta}-\theta^{*}\right\| \leqslant\left\|\tilde{\theta}-\hat{\theta}_{0}\right\|+\left\|\hat{\theta}_{0}-\theta^{*}\right\| \leqslant \frac{A_{0}}{A_{0}+A_{1}} L+\left\|\hat{\theta}_{0}-\theta^{*}\right\| .
$$

Известно (см. [3]), что для обычной ОМП $\breve{\theta}_{0}$ справедливо равенство

$$
\lim _{n \rightarrow \infty} \mathbf{P}_{\theta^{*}, 0}\left\{\sqrt{n}\left\|\breve{\theta}_{0}-\theta^{*}\right\| \geqslant x\right\}=C \exp \left(-C x^{2}\right) .
$$

Отсюда для СОМП $\hat{\theta}_{0}$ получаем

$$
\lim _{n \rightarrow \infty} \mathbf{P}_{\theta^{*}, 0}\left\{\sqrt{n}\left\|\hat{\theta}_{0}-\theta^{*}\right\| \geqslant x\right\} \leqslant C \exp \left(-C x^{2}\right) .
$$

Заметим также, что если $z<\delta / 2(\delta$ из условия $(2))$, то имеет место включение событий $\left\{\operatorname{dis}\left(\hat{\theta}_{1}, \Theta\right) \leqslant z\right\} \subset\left\{\operatorname{dis}\left(\hat{\theta}_{1}, Z_{1}\right)>z\right\}$. 
Поэтому, используя условие (2) и оценку (7), получаем для любого (фиксированного) $x>0$ :

$$
\begin{aligned}
\mathbf{P}_{\theta^{*}, 0} & \left\{\sqrt{n}\left\|\tilde{\theta}-\hat{\theta}_{0}\right\|>\frac{x}{2}\right\} \leqslant \mathbf{P}_{\theta^{*}, 0}\left\{\frac{\sqrt{n} A_{0}}{A_{0}+A_{1}}>\frac{x}{2 L}\right\} \\
= & \mathbf{P}_{\theta^{*}, 0}\left\{\frac{\sqrt{n} A_{0}}{A_{0}+A_{1}}>\frac{x}{2 L}, \operatorname{dis}\left(\hat{\theta}_{1}, \Theta\right)>\frac{\delta}{4}\right\} \\
& +\mathbf{P}_{\theta^{*}, 0}\left\{\frac{\sqrt{n} A_{0}}{A_{0}+A_{1}}>\frac{x}{2 L}, \operatorname{dis}\left(\hat{\theta}_{1}, \Theta\right) \leqslant \frac{\delta}{4}\right\} \\
\leqslant & \mathbf{P}_{\theta^{*}, 0}\left\{\sqrt{n} \operatorname{dis}\left(\hat{\theta}_{0}, \Theta\right)>\frac{x \delta}{8 L}\right\}+\mathbf{P}_{\theta^{*}, 0}\left\{\operatorname{dis}\left(\hat{\theta}_{1}, \Theta\right) \leqslant \frac{\delta}{4}\right\} \\
\leqslant & \mathbf{P}_{\theta^{*}, 0}\left\{\sqrt{n} \operatorname{dis}\left(\hat{\theta}_{0}, \Theta\right)>\frac{x \delta}{8 L}\right\}+\mathbf{P}_{\theta^{*}, 0}\left\{\operatorname{dis}\left(\hat{\theta}_{1}, Z_{1}\right)>\frac{\delta}{4}\right\} \\
\leqslant & (1+o(1))\left(C \exp \left(-C x^{2} \delta^{2}\right)+o(1)\right)=(1+o(1)) C \exp \left(-C x^{2}\right) .
\end{aligned}
$$

Теперь утверждение леммы для $i=0$ следует из (6) и (7). Для $i=1$ доказательство проводится аналогично.

Положим

$$
J=\ln \frac{f_{1}(\tilde{X}, \tilde{\theta})}{f_{0}(\tilde{X}, \tilde{\theta})}, \quad c^{*}=\ln \frac{\beta}{\alpha}
$$

и рассмотрим следующее решающее правило:

$$
\begin{array}{llll}
\text { на множестве } & \widehat{D}_{1}=\left(\tilde{X}: J>c^{*}\right\} & \text { принимается } & \mathbf{H}_{1} ; \\
\text { на множестве } & \widehat{D}_{0}=\left\{\tilde{X}: J \leqslant c^{*}\right\} & \text { принимается } & \mathbf{H}_{0} .
\end{array}
$$

Обозначим через $\varepsilon_{i}^{\star}(\widetilde{n}), i=0,1$, соответствующие вероятности ошибок для оптимального байесовского правила при известном значении параметра $\theta^{*}$ и использовании части наблюдений $\widetilde{X}$, т.е. $\varepsilon_{1}^{\star}(\widetilde{n})=$ $\mathbf{P}\left\{D_{1} \mid \mathbf{H}_{0}, \theta=\theta^{\star}\right\}, \varepsilon_{0}^{\star}(\widetilde{n})=\mathbf{P}\left\{D_{0} \mid \mathbf{H}_{1}, \theta=\theta^{\star}\right\}$.

Введем логарифм отношения правдоподобия

$$
\lambda(x, \theta)=\ln \frac{f_{1}(x, \theta)}{f_{0}(x, \theta)} .
$$

Теорема 1. Пусть для любого $\theta \in \Theta$ u $i=0,1$ выполнены следующие условия:

а) функция $\lambda(x, \theta)$ непрерывно дифференцируема по $\theta$ и существует положительная функиия $K(x)$ такая, что

$$
\begin{gathered}
\sup _{\theta}\left\|\lambda_{\theta}^{\prime}(x, \theta)\right\| \leqslant K(x), \\
\mathbf{E}_{\theta, i} K(u) \triangleq m_{i}=\int K(u) f_{i}(u, \theta) d \mu(u)<\infty, \\
\mathbf{P}_{\theta, i}\left\{\left|K(u)-m_{i}\right| \geqslant z\right\} \leqslant C \exp (-C z) \quad \forall z>0 ;
\end{gathered}
$$


b) оба распределения $\mathbf{P}_{\theta, 0}, \mathbf{P}_{\theta, 1}$ случайной величинь $\lambda(x, \theta)$ имеют абсолютно непрерывную компоненту;

c) $\mathbf{E}_{\theta, i} \exp (\gamma \lambda(z, \theta))<\infty$ для некоторого $\gamma>0$.

Тогда при любом выборе $\tilde{n}<n$, для которого $\tilde{n} / n \rightarrow 0$ nри $n \rightarrow \infty$, $\tilde{n} \rightarrow \infty$, решающее правило (8) асимптотически эквивалентно оптимальному байесовскому правилу для любого известного значения параметра в следуюшем смысле:

$$
\lim _{n \rightarrow \infty} \frac{\ln \mathbf{P}_{\theta^{*}, 0}\left(\widehat{D}_{1}\right)}{\ln \varepsilon_{1}^{*}(\widetilde{n})}=1, \quad \lim _{n \rightarrow \infty} \frac{\ln \mathbf{P}_{\theta^{\star}, 1}\left(\widehat{D}_{0}\right)}{\ln \varepsilon_{0}^{\star}(\widetilde{n})}=1
$$

для любого $\theta^{\star} \in \Theta$.

Д о к а з а т е л с с т о. Рассмотрим первое равенство в (9) и будем для упрощения записи использовать символ $\mathbf{P}$ вместо $\mathbf{P}_{\theta^{*}, 0}$.

Вероятность ошибочного решения для правила (8) можно записать в виде

$$
\mathbf{P}\left(\widehat{D}_{1}\right)=\mathbf{P}\left\{\frac{1}{\widetilde{n}} \sum_{i=1}^{\tilde{n}} \zeta_{i}(\tilde{\theta})>c_{\widetilde{n}}\right\}
$$

где $\zeta_{i}(\theta) \triangleq \lambda\left(x_{i}, \theta\right), c_{\widetilde{n}}=\tilde{n}^{-1} c^{*}$.

$\mathrm{B}$ силу условия а) имеем

$$
\zeta_{i}(\tilde{\theta})=\zeta_{i}\left(\theta^{\star}\right)+\left\langle r\left(x_{i}, \theta\right), \tilde{\theta}-\theta^{*}\right\rangle,
$$

где $\sup _{\theta}\left\|r\left(x_{i}, \theta\right)\right\| \leqslant K\left(x_{i}\right)$.

Так как $0<A \triangleq-\mathbf{E}_{\theta^{\star}, 0} \lambda\left(\cdot, \theta^{\star}\right)$, нам требуется оценить такую вероятность:

$$
\begin{aligned}
S & \triangleq \mathbf{P}\left\{\widetilde{n}^{-1} \sum_{i=1}^{\widetilde{n}} \xi_{i}+\widetilde{n}^{-1} \sum_{i=1}^{\widetilde{n}}\left\langle r\left(x_{i}, \theta\right), \tilde{\theta}-\theta^{*}\right\rangle>c_{\widetilde{n}}+A\right\} \\
& \triangleq \mathbf{P}\left\{U_{\widetilde{n}}+V_{\widetilde{n}}>c_{\widetilde{n}}+A\right\}
\end{aligned}
$$

где $\mathbf{E} \xi_{i}=0$.

Так как $\left|V_{\widetilde{n}}\right| \leqslant \widetilde{n}^{-1}\left\|\tilde{\theta}-\theta^{*}\right\| \sum_{i=1}^{\widetilde{n}} K\left(x_{i}\right) \triangleq \mathscr{Z}_{\widetilde{n}}$ и $(1 \geqslant \varepsilon \geqslant 0)$

$$
\begin{aligned}
& \mathbf{P}\left\{U_{\widetilde{n}}>(1+\varepsilon)\left(c_{\widetilde{n}}+A\right)\right\}-\mathbf{P}\left\{\mathscr{Z}_{\widetilde{n}}>\varepsilon\left(c_{\widetilde{n}}+A\right)\right\} \\
& \quad \leqslant S \leqslant \mathbf{P}\left\{U_{\widetilde{n}}>(1-\varepsilon)\left(c_{\widetilde{n}}+A\right)\right\}+\mathbf{P}\left\{\mathscr{Z}_{\widetilde{n}}>\varepsilon\left(c_{\widetilde{n}}+A\right)\right\},
\end{aligned}
$$

то достаточно изучить поведение вероятности

$$
Q=\mathbf{P}\left\{\left\|\tilde{\theta}-\theta^{*}\right\| \tilde{n}^{-1} \sum_{i=1}^{\tilde{n}} K\left(x_{i}\right)>x\right\}, \quad x>0 .
$$

Положим

$$
Z_{\widetilde{n}}=\sum_{i=1}^{\tilde{n}}\left(K\left(x_{i}\right)-m\right), \quad \eta_{\widetilde{n}}=\tilde{n}^{-1} Z_{\widetilde{n}}
$$


где $m=\mathbf{E} K\left(x_{i}\right)>0$. Тогда для любого $N$ такого, что $\sqrt{n} x /(2 m)>$ $N>0$, получаем

$$
\begin{aligned}
Q & =\mathbf{P}\left\{\left\|\tilde{\theta}-\theta^{*}\right\|\left(\eta_{\widetilde{n}}+m\right) \sqrt{n}>\sqrt{n} x\right\} \\
& \leqslant 2 \mathbf{P}\left\{\sqrt{n}\left\|\tilde{\theta}-\theta^{*}\right\|>N\right\}+\mathbf{P}\left\{\eta_{\widetilde{n}}>\frac{\sqrt{n} x}{2 N}\right\} \\
& =2 \mathbf{P}\left\{\sqrt{n}\left\|\tilde{\theta}-\theta^{*}\right\|>N\right\}+\mathbf{P}\left\{Z_{\widetilde{n}}>\frac{\sqrt{n} x \widetilde{n}}{2 N}\right\} .
\end{aligned}
$$

Для оценки второго слагаемого в (13) воспользуемся экспоненциальным неравенством для сумм независимых случайных величин $([4$, с. 81$])$, которое справедливо в силу предположения а):

$$
\mathbf{P}\left\{Z_{\widetilde{n}}>\frac{\sqrt{n} x \widetilde{n}}{2 N}\right\} \leqslant \exp \left(-C \frac{\sqrt{n} x \widetilde{n}}{N}\right) .
$$

В силу леммы 1 и неравенств (13) и (14) получаем, что при $\sqrt{n} x(2 m)^{-1}>N>0$

$$
Q \leqslant C\left(\exp \left(-C N^{2}\right)+\exp \left(-C \frac{\tilde{n} x \sqrt{n}}{N}\right)\right) .
$$

Пусть $\widetilde{n} / n \rightarrow 0$ при $n \rightarrow \infty, \widetilde{n} \rightarrow \infty$. Тогда точка минимума по $N$ в правой части (15), $N=O\left((x \widetilde{n} \sqrt{n})^{1 / 3}\right)$, при достаточно больших $n$ попадет в интервал $\left(0, \sqrt{n} x(2 m)^{-1}\right)$ и поэтому из $(15)$ получаем

$$
Q \leqslant C \exp \left(-C(\widetilde{n} \sqrt{n} x)^{2 / 3}\right) .
$$

Далее мы можем воспользоваться техническими условиями b)-c), из которых следует (см. [3, с. 289]), что

$$
\begin{aligned}
& \lim _{\tilde{n} \rightarrow \infty} \widetilde{n}^{-1} \ln \varepsilon_{1}^{\star}=\lim _{\tilde{n} \rightarrow \infty} \widetilde{n}^{-1} \ln \mathbf{P}\left\{U_{\widetilde{n}}>A+c_{\widetilde{n}}\right\}=-A, \\
& \lim _{\tilde{n} \rightarrow \infty} \widetilde{n}^{-1} \ln \mathbf{P}\left\{U_{\widetilde{n}}>(1 \pm \varepsilon)\left(A+c_{\widetilde{n}}\right)\right\}=-(1 \pm \varepsilon) A+o(\varepsilon), \quad \varepsilon \rightarrow 0 .
\end{aligned}
$$

Для любого $\varepsilon>0$ можно указать такое $n_{0}$, что при $n>n_{0}$ будет выполняться условие $(\sqrt{n}(\varepsilon A) \widetilde{n})^{2 / 3}>(1+\varepsilon) A \widetilde{n}$ что, в свою очередь, в силу $(12),(16)$ и $(17)$, приводит к соотношению

$$
\begin{aligned}
& (1+o(1)) \exp \{(-A(1+\varepsilon)+o(\varepsilon)) \widetilde{n}\} \\
& \quad \leqslant S \leqslant(1+o(1)) \exp \{(-A(1-\varepsilon)+o(\varepsilon)) \widetilde{n}\} .
\end{aligned}
$$

Отсюда

$$
1+\varepsilon+o(\varepsilon) \geqslant \lim _{\tilde{n} \rightarrow \infty} \frac{\ln \mathbf{P}\left\{U_{\widetilde{n}}+V_{\widetilde{n}}>c_{\widetilde{n}}+A\right\}}{\ln \mathbf{P}\left\{U_{\widetilde{n}}>c_{\widetilde{n}}+A\right\}} \geqslant 1-\varepsilon+o(\varepsilon),
$$

что, в силу произвольности $\varepsilon$, доказывает первое равенство в (9). Аналогичные рассмотрения можно провести и для второй меры (второго равенства) в (9).

Теорема доказана. 
3 а м е ч а н и е 1 . В условии (2) можно использовать проекцию на шар $K$ произвольной асимптотически нормальной оценки параметра $\theta$ вместо ОМП.

3 а м е ч а н и е 2. Допустим, что существует такая оценка параметра, для которой справедливы соотношения (5). Тогда такую оценку можно использовать в решающем правиле (8), и при этом условие (2) будет лишним. В частности, так обстоит дело, если неизвестный параметр - это параметр сдвига для обеих плотностей, допускающий асимптотически нормальную оценку.

3. Второе решающее правило. Рассмотрим теперь другой способ решения нашей проблемы.

В этом пункте мы будем предполагать (помимо условий 1)-3)), что плотности $f_{i}$ существуют по отношению к лебеговой мере.

Фиксируем $s>0$ и зададим на множестве $\Theta_{s}$ вероятностные меры с плотностями $\mathfrak{m}_{i}(\theta \mid X), i=0,1$, следующим образом:

$$
\mathfrak{m}_{i}(\theta \mid X) \triangleq \mathfrak{m}_{i}(\theta)= \begin{cases}0, & \text { если } \theta \notin \Theta_{s}, \\ \frac{f_{i}(X, \theta)}{\int_{\Theta_{s}} f_{i}(X, \theta) d \theta}, & \text { если } \theta \in \Theta_{s}, \quad i=0,1 .\end{cases}
$$

Легко видеть, что $\mathfrak{m}_{i}(\theta)$ есть апостериорные плотности параметра, при условии, что наблюдена выборка $X$, если предположить, что $\theta-$ случайная величина с равномерным априорным распределением на множестве $\Theta_{s}$. Можно, однако, использовать эти плотности без такого предположения и даже для неограниченного множества $\Theta$. Смысл введения подобных «апостериорных» мер состоит в том, что они задают естественный уровень доверия к точкам множества $\Theta$ после получения наблюдений. Отметим, что введение подобных мер предлагалось з [5] при рассмотрении стохастической задачи восстановления.

Хорошо известно (см., например, [6], [7]), что при условиях регулярности апостериорная плотность случайной величины $\sqrt{N}\left(\theta-\hat{\theta}_{N}\right)$ равномерно на любом компакте сходится по вероятности к плотности нормального распределения с нулевым средним и некоторой конечной матрицей ковариаций при $N \rightarrow \infty$ (здесь $N$ - объем выборки и $\hat{\theta}_{N}-$ ОМП параметра $\theta$ ). Поэтому, если $g(\theta)$ - непрерывная и ограниченная функция, то

$$
\int_{\Theta} g(\theta) p_{N}(\theta \mid \cdot) d \theta \stackrel{\mathbf{P}_{\theta^{*}}}{\longrightarrow} g\left(\theta^{*}\right),
$$

где $\theta^{*} \in \Theta$ есть истинное значение параметра и $p_{N}(\theta \mid \cdot)-$ апостериорная плотность.

Соотношение (18) позволяет предложить новое решающее правило для рассматриваемой нами проблемы, основанное на плотностях $\mathfrak{m}_{i}(\theta)$, $i=0,1$. 
Положим

$$
\theta_{i}^{a p}=\int_{\Theta_{s}} \theta \mathfrak{m}_{i}(\theta) d \theta, \quad i=0,1
$$

и введем оценку

$$
\tilde{\theta}^{A P}=\frac{A_{1}}{A_{0}+A_{1}} \theta_{0}^{a p}+\frac{A_{0}}{A_{0}+A_{1}} \theta_{1}^{a p},
$$

где $A_{0}, A_{1}$ определены в (4).

Введем функцию (информация Кульбака-Лейблера)

$$
K_{i}\left(\theta^{*}, \theta\right)=\int\left(\ln \frac{f_{i}\left(x, \theta^{*}\right)}{f_{i}(x, \theta)}\right) f_{i}\left(x, \theta^{*}\right) d x, \quad i=0,1 .
$$

Лемма 2. Пусть выполнены сделанные выше предположения и, кроме того, Функции $K_{i}\left(\theta^{*}, \theta\right)$ дважды непрерьвно дифференцируемь no $\theta$.

Тогда существует положительная константа $C$ такая, что для любых $\theta^{*} \in \Theta u i=0,1$

$$
\left\|\tilde{\theta}^{A P}-\theta^{*}\right\| \leqslant C \sqrt{n^{-1} \ln n}+\xi_{n} \quad \mathbf{P}_{\theta^{*}, i}-\text { n.н. },
$$

где при всех $x>0$ для случайнойєвеличины $\xi_{n}$ справедливы соотношения

$$
\lim _{n \rightarrow \infty} \mathbf{P}_{\theta^{*}, i}\left\{\sqrt{n} \xi_{n}>x\right\} \leqslant C \exp \left(-C x^{2}\right), \quad i=0,1 .
$$

Д о к а з а т е л ь с т в о. Проведем доказательство для меры $\mathbf{P}_{\theta^{*}, 0}$, опуская соответствующие индексы.

Воспользуемся результатами работы [8]. Из них следует, что условие дифференцируемости функций $K_{i}(\cdot)$ в нашей ситуации гарантирует существование предела

$$
\lim _{n \rightarrow \infty} n^{-1} \ln \left(\int_{\|\theta-\breve{\theta}\|>z} \mathfrak{m}(\theta) d \theta\right)=-\min _{\|h\|=1}\left\{K\left(\theta^{*}, \breve{\theta}+z h\right)\right\} \quad \text { P-п.н., }
$$

где $\breve{\theta}$ - ОМП (на самом деле, в [8] рассматривается скалярный параметр, однако это связано с тем, что, усиливая свои прежние результаты, авторы изучают некомпактное параметрическое множество, из-за чего потребовалось использовать негладкую нормализующую функцию; в компактном случае нам достаточен прежний результат, цитируемый авторами в [8] и справедливый, как подтвердили авторы в частном сообщении, для многомерного параметра).

Известно (см. [3, с. 201]), что в условиях регулярности имеет место оценка $(\|h\|=1): K\left(\theta^{*}, \breve{\theta}+z h\right) \geqslant C\left\|\left(\breve{\theta}-\theta^{*}\right)+z h\right\|^{2} \geqslant C\left|\left\|\breve{\theta}-\theta^{*}\right\|-z\right|^{2}$.

Поэтому из (19) получаем

$$
\int_{\|\theta-\breve{\theta}\|>z} \mathfrak{m}(\theta) d \theta \leqslant \exp \left(-C n\left|\left\|\breve{\theta}-\theta^{*}\right\|-z\right|^{2}\right),
$$

откуда следует, что для $z>0$

$$
\left\|\theta^{a p}-\breve{\theta}\right\| \leqslant z+\exp \left(-C n\left|\left\|\breve{\theta}-\theta^{*}\right\|-z\right|^{2}\right) \quad \text { Р-п.н. }
$$


Выбирая в $(20) z$ из условия ||$\breve{\theta}-\theta^{*} \|-z \mid=\sqrt{(\ln n) /(C n)}$, получаем, что

$$
\left\|\theta^{a p}-\breve{\theta}\right\| \leqslant C \sqrt{\frac{\ln n}{n}}+\left\|\breve{\theta}-\theta^{*}\right\| \quad \text { Р-п.н. }
$$

Таким образом, Р-п.н.

$$
\begin{aligned}
\left\|\tilde{\theta}^{A P}-\theta^{*}\right\| & =\left\|\theta_{0}^{a p}-\breve{\theta}+\breve{\theta}-\theta^{*}+\left(\theta_{1}^{a p}-\theta_{0}^{a p}\right) \frac{A_{0}}{A_{0}+A_{1}}\right\| \\
& \leqslant C \sqrt{\frac{\ln n}{n}}+\frac{A_{0} L}{A_{0}+A_{1}}+2\left\|\breve{\theta}-\theta^{*}\right\|,
\end{aligned}
$$

где для случайной величины $\xi_{n} \triangleq A_{0} L /\left(A_{0}+A_{1}\right)+2\left\|\breve{\theta}-\theta^{*}\right\|$ экспоненциальное неравенство вытекает из свойств ОМП и леммы 1.

Аналогичные рассуждения проводятся и для второй меры. Лемма доказана.

Положим

$$
I=\ln \frac{f_{1}\left(\tilde{X}, \tilde{\theta}^{A P}\right)}{f_{0}\left(\widetilde{X}, \tilde{\theta}^{A P}\right)}
$$

и рассмотрим следуюшее решающее правило:

на множестве $\widetilde{D}_{1}=\left\{\tilde{X}: I>c^{*}\right\}$ принимается $\mathbf{H}_{1}$;

на множестве $\widetilde{D}_{0}=\left\{\tilde{X}: I \leqslant c^{*}\right\}$ принимается $\mathbf{H}_{0}$.

Теорема 2. Пусть для любого $\theta \in \Theta$ и $i=0,1$ выполнены следующие условия:

а) Функиия $\lambda(x, \theta)$ непрерьвно дифференцируема по $\theta$ и существует положительная функиия $G(x)$ такая, что

$$
\begin{gathered}
\sup _{\theta}\left\|\lambda_{\theta}^{\prime}(x, \theta)\right\| \leqslant G(x), \\
\mathbf{E}_{\theta, i} G(u) \triangleq m_{i}=\int G(u) f_{i}(u, \theta) d u<\infty, \\
\mathbf{P}_{\theta, i}\left\{\left|G(u)-m_{i}\right| \geqslant z\right\} \leqslant C \exp (-C z) \quad \forall z>0 ;
\end{gathered}
$$

b) оба распределения $\mathbf{P}_{\theta, 0}, \mathbf{P}_{\theta, 1}$ случайной величины $\lambda(x, \theta)$ имеют абсолютно непрерывную компоненту;

c) $\mathbf{E}_{\theta, i} \exp (\gamma \lambda(z, \theta))<\infty$ для некоторого $\gamma>0$;

d) Функции $K_{i}\left(\theta^{*}, \theta\right)$ дважды непрерьвно дифференцируемьи по $\theta$.

Тогда, если $\tilde{n} / n \rightarrow 0$ при $n \rightarrow \infty, \tilde{n} \rightarrow \infty$, то решающее правило (23) асимптотически эквивалентно оптимальному байесовскому правилу для любого известного значения параметра в следуюшем смьcлe:

$$
\lim _{n \rightarrow \infty} \frac{\ln \mathbf{P}_{\theta^{*}, 0}\left(\widetilde{D}_{1}\right)}{\ln \varepsilon_{1}^{*}(\widetilde{n})}=1, \quad \lim _{n \rightarrow \infty} \frac{\ln \mathbf{P}_{\theta^{\star}, 1}\left(\widetilde{D}_{0}\right)}{\ln \varepsilon_{0}^{\star}(\widetilde{n})}=1
$$

для любого $\theta^{\star} \in \Theta$. 
Д ок а з а т е л с с в о. Рассмотрим первое равенство в (24) и будем для упрощения записи использовать символ $\mathbf{P}$ вместо $\mathbf{P}_{\theta^{*}, 0}$.

Доказательство проводится по той же схеме, что и в теореме 1 с той разницей, что теперь оценка для $V_{\widetilde{n}}$ из соотношения (11) имеет, в силу леммы 2, Р-п.н. следующий вид:

$$
|V|_{\widetilde{n}} \leqslant\left(C \sqrt{n^{-1} \ln n}+\xi_{n}\right) \tilde{n}^{-1} \sum_{i=1}^{\widetilde{n}} G\left(x_{i}\right),
$$

где случайная величина $\xi_{n}$ определена в лемме 2 .

Оценка вероятности уклонения от нуля для второго слагаемого в правой части (25) проводилась в доказательстве теоремы 1. Для первого слагаемого имеем

$$
\begin{aligned}
& \mathbf{P}\left\{C \sqrt{n^{-1} \ln n} \tilde{n}^{-1} \sum_{i=1}^{\tilde{n}}\left(G\left(x_{i}\right)-m\right)>z-C m \sqrt{n^{-1} \ln n}\right\} \\
& \quad \leqslant \mathbf{P}\left\{C \sqrt{n^{-1} \ln n} \tilde{n}^{-1} \sum_{i=1}^{\tilde{n}}\left(G\left(x_{i}\right)-m\right)>\frac{z}{2}\right\}
\end{aligned}
$$

если $n$ достаточно велико. Поэтому, вновь пользуясь экспоненциальным неравенством для сумм независимых случайных величин, устанавливаем, что поведение вероятности уклонения от нуля для $V_{\widetilde{n}}$ определяется вторым слагаемым в правой части (25) и, следовательно, дальнейшие рассуждения можно провести точно так же, как и при доказательстве теоремы 1.

З а м е ч ан и е 3 . Известно (см. [3, с. 245]), что байесовская оценка параметра в условиях регулярности асимптотически эквивалентна ОМП для любой априорной плотности, которая положительна и непрерывна на множестве $\Theta$. В нашем случае это означает, что $\sqrt{n}\left(\hat{\theta}_{i}-\theta_{i}^{a p}\right) \longrightarrow 0 \mathbf{P}_{\theta^{*}, i}$-П.н., $i=0,1$.

Поэтому, если положить $\Theta_{s}=K$ (напомним, что $K$ - это шар, использовавшийся при построении (ОМП), то в описанном выше решающем правиле (23) можно заменить СОМП в формулах для $A_{i}$ на $\theta_{i}^{a p}$, имея в виду, что последние гораздо легче вычисляются.

4. Задача с двумя параметрическими множествами. Подход, описанный в п. 3, дает возможность предложить новое решаюшее правило и для задачи проверки следующих гипотез:

$$
\begin{aligned}
& \widetilde{\mathbf{H}}_{1} \text { : совместная плотность наблюдений равна } f(X, \theta), \theta \in \Theta_{1}, \\
& \tilde{\mathbf{H}}_{0} \text { : совместная плотность наблюдений равна } f(X, \theta), \theta \in \Theta_{0},
\end{aligned}
$$
где $\theta-$-еизвестньй параметр. 
Предположим, что

$$
\begin{gathered}
\Theta_{0} \cap \Theta_{1}=\varnothing, \\
f\left(\cdot, \theta_{1}\right) \neq f\left(\cdot, \theta_{2}\right) \quad \text { на множестве }
\end{gathered}
$$

положительной лебеговой меры, если $\theta_{1} \neq \theta_{2}$.

Очевидно, что если выполнено (26), то выполнено и условие детектируемости, аналогичное (1).

Рассмотрим произвольную пару $\left(\theta_{0}, \theta_{1}\right)$ такую, что $\theta_{0} \in \Theta_{0}, \theta_{1} \in \Theta_{1}$. Обозначим через $\mathbf{P}_{\theta_{0}}\left(\mathbf{P}_{\theta_{1}}\right)$ вероятностную меру, соответствующую значению параметра $\theta_{0}\left(\theta_{1}\right)$.

Положим $\Theta=\Theta_{0} \cup \Theta_{1}$. Аналогично п. 2, мы будем предполагать, что множество $\Theta$ - компакт, для всех $\theta$ из некоторой окрестности $\Theta$ выполнены условия регулярности плотности $f(x, \theta)$ и справедливо условие (26).

Рассмотрим $\delta$-окрестность $\Theta$ и зададим вероятностную плотность $\mathfrak{m}(\theta)$ («апостериорную» плотность) на множестве $\Theta_{\delta}$ так же, как в п. 3 , но для одной плотности $f$. Положим

$$
\theta^{A P}=\int_{\Theta_{\delta}} \theta \mathfrak{m}(\theta) d \theta, \quad \theta_{0}^{A P}=\operatorname{Pr}_{\Theta_{0}} \theta^{A P}, \quad \theta_{1}^{A P}=\operatorname{Pr}_{\Theta_{1}} \theta^{A P},
$$

где $\operatorname{Pr}_{M}$ - оператор проектирования на множество $M$.

Рассмотрим следующий критерий:

$$
\tilde{J}=\ln \frac{f\left(\tilde{X}, \theta_{1}^{A P}\right)}{f\left(\widetilde{X}, \theta_{0}^{A P}\right)}
$$

где, напомним, $\widetilde{X}=\left\{x_{1}, \ldots, x_{\widetilde{n}}\right\}, \widetilde{n}<n$.

Предлагаемое решающее правило имеет вид (напомним, что $c^{*}=$ $\ln (\beta / \alpha))$ :

$$
\begin{array}{llll}
\text { если } & \tilde{J} \leqslant c^{*}, & \text { принять } & \tilde{\mathbf{H}}_{0}, \\
\text { если } & \tilde{J}>c^{*}, & \text { принять } & \widetilde{\mathbf{H}}_{1} .
\end{array}
$$

Если величины $\theta_{0}, \theta_{1}$ известны, то оптимальное байесовское правило для задачи (3) на основе выборки $\widetilde{X}$ имеет вид:

$$
\begin{array}{ll}
\text { если } & \ln \frac{f\left(\tilde{X}, \theta_{1}\right)}{f\left(\widetilde{X}, \theta_{0}\right)} \leqslant c^{*}, \quad \text { принять } \widetilde{\mathbf{H}}_{0}, \\
\text { если } & \ln \frac{f\left(\widetilde{X}, \theta_{1}\right)}{f\left(\widetilde{X}, \theta_{0}\right)}>c^{*}, \quad \text { принять } \widetilde{\mathbf{H}}_{1} .
\end{array}
$$

Обозначим

$$
\begin{aligned}
& \varepsilon_{1}\left(\theta_{0}, \theta_{1}, \tilde{n}\right)=\mathbf{P}_{\theta_{0}}\left\{\ln \frac{f\left(\tilde{X}, \theta_{1}\right)}{f\left(\tilde{X}, \theta_{0}\right)}>c^{*}\right\}, \\
& \varepsilon_{0}\left(\theta_{0}, \theta_{1}, \tilde{n}\right)=\mathbf{P}_{\theta_{1}}\left\{\ln \frac{f\left(\tilde{X}, \theta_{1}\right)}{f\left(\tilde{X}, \theta_{0}\right)} \leqslant c^{*}\right\}
\end{aligned}
$$

соответствующие вероятности ошибок для правила (28). 
Для любых $\theta=\left(\theta_{0}, \theta_{1}\right), \theta_{i}^{*} \in \Theta_{i}, i=0,1$, введем обозначения

$$
\begin{aligned}
\lambda(x, \theta) & =\ln \frac{f\left(x, \theta_{1}\right)}{f\left(x, \theta_{0}\right)}, \\
K\left(\theta_{i}^{*}, \theta_{i}\right) & =\int\left(\ln \frac{f\left(x, \theta_{i}^{*}\right)}{f\left(x, \theta_{i}\right)}\right) f\left(x, \theta_{i}^{*}\right) d x, \quad i=0,1 .
\end{aligned}
$$

Тогда, аналогично теореме 2 и учитывая, что оценка $\theta^{A P}$ асимптотически эквивалентна ОМП, можно установить, что верна следующая теорема.

Теорема 3. Пусть для $i=0,1$ и любьх $\theta_{i}^{*} \in \Theta_{i}$ выполнень следующие условия:

а) Функиия $\lambda(x, \theta)$ непрерьвно дифференцируема по $\theta$, и существует положительная функиия $R(x)$ такая, что

$$
\begin{gathered}
\sup _{\theta \in \Theta_{\delta}}\left\|\lambda_{\theta}^{\prime}(x, \theta)\right\| \leqslant R(x), \\
\mathbf{E}_{\theta_{i}^{*}} R(u) \triangleq m_{i}=\int R(u) f\left(u, \theta_{i}^{*}\right) d u<\infty, \\
\mathbf{P}_{\theta_{i}^{*}}\left\{\left|R(u)-m_{i}\right| \geqslant z\right\} \leqslant C \exp (-C z) \quad \forall z>0 ;
\end{gathered}
$$

b) оба распределения $\mathbf{P}_{\theta_{0}^{*}}, \mathbf{P}_{\theta_{1}^{*}}$ случайной величины $\lambda\left(x, \theta^{*}\right)$ имеют абсолютно непрерьвную компоненту;

c) $\mathbf{E}_{\theta_{i}^{*}} \exp \left(\gamma \lambda\left(z, \theta^{*}\right)\right)<\infty$ для некоторого $\gamma>0$;

d) Функиии $K\left(\theta_{i}^{*}, \theta_{i}\right)$ дважды непрерьвно диФференцируемь по $\theta_{i}$.

Тогда, если $\tilde{n} / n \rightarrow 0$ рри $n \rightarrow \infty, \tilde{n} \rightarrow \infty$, то решающее правило (28) асимптотически эквивалентно оптимальному байесовскому правилу для любых известных значений $\theta_{0}^{*} \in \Theta_{0}, \theta_{1}^{*} \in \Theta_{1}$ при соответствуюших альтернативах $\phi_{1}\left(\theta_{0}^{*}\right)=\operatorname{Pr}_{\Theta_{1}} \theta_{0}^{*}, \phi_{0}\left(\theta_{1}^{*}\right)=\operatorname{Pr}_{\Theta_{0}} \theta_{1}^{*}$ в следуюшем смисле:

$$
\lim _{n \rightarrow \infty} \frac{\ln \mathbf{P}_{\theta_{0}^{*}}\left\{\tilde{J}>c^{*}\right\}}{\ln \varepsilon_{1}\left(\theta_{0}^{*}, \phi_{1}\left(\theta_{0}^{*}\right), \tilde{n}\right)}=1, \quad \lim _{n \rightarrow \infty} \frac{\ln \mathbf{P}_{\theta_{1}^{*}}\left\{\tilde{J} \leqslant c^{*}\right\}}{\ln \varepsilon_{0}\left(\phi_{0}\left(\theta_{1}^{*}\right), \theta_{1}^{*}, \tilde{n}\right)}=1 .
$$

3 а м е ч а н и е 4. Рассмотренные в статье два метода проверки гипотез можно сравнивать между собой по следующим критериям: a) соотношение между $\widetilde{n}$ и $n$; b) сложность вычислений; с) спектр решаемых задач.

Как можно увидеть из доказательств, скорость сходимости к пределу отношения логарифмов вероятностей ошибок для первого метода выше, чем для второго при том же соотношении между $\tilde{n}$ и $n$. Однако ОМП, необходимая для первого метода, может потребовать сложных вычислений. С другой стороны, второй метод может быть использован для решения как исходной задачи, так и задачи, описанной в п. 4, в то время как первый метод применим только для задачи п. 1. Поэтому, на 
наш взгляд, для моделей с одним параметрическим множеством и простой процедурой вычисления ОМП удобно применять первый метод, а во всех остальных случаях (если нет серьезных вычислительных ограничений) - второй.

3 а м е ч а н и е 5. Если в предлагаемых методах положить $\tilde{n}=n$, то для задачи с двумя параметрическими множествами фактически получаем известное эвристическое правило, а для задачи с одним параметрическим множеством - его естественный аналог. Тем самым, предлагаемые методы заведомо не хуже широко используемых эвристик (как уже известных, так и их потенциальных аналогов), но в то же время позволяют вести осмысленный поиск методов, более эффективных, чем известные. Кроме того, применение предложенной здесь «апостериорной» оценки вместо ОМП позволяет существенно упростить также и известный эвристический метод.

Первый вариант статьи детально обсуждался с Д. М. Чибисовым. В результате дискуссии были устранены серьезные недостатки, а рукопись подверглась радикальной переработке. Мы весьма признательны Д. М. Чибисову за эту дискуссию.

Авторы глубоко благодарны Э.В. Хмаладзе и Ю.А. Давыдову за внимание к работе и выражают признательность Я. Ю. Никитину, указавшему статью [8].

\section{СПИСОК ЛИТЕРАТУРЫ}

1. Bahadur R. R. An optimal property of the likelihood ratio statistic. - Proceedings of the Fifth Berkeley Symposium on Mathematical Statistics and Probability, University of California, 1965/1966. V. 1. 1966, p. 13-26.

2. Brown L. D. Non-local asymptotic optimality of appropriate likelihood ratio tests. Ann. Math. Statist., 1971, v. 42, № 4, p. 1206-1240.

3. Боровков А. А. Математическая статистика. М.: Наука, 1984, 472 с.

4. Петров В. В. Предельные теоремы для сумм независимых случайных величин. М.: Наука, 1987, 317 с.

5. Дарховский Б. С. О стохастической задаче восстановления. - Теория вероятн. и ее примен., 1998, т. 43, в. 2, с. 357-364.

6. Walker A.M. On the asymptotic behaviour of posterior distributions. - J. Roy. Statist. Soc., Ser. B, 1969, v. 31, p. 80-88.

7. Schervish M. J. Theory of Statistics. Berlin-Heidelberg: Springer-Verlag, 1995, 702 p.

8. Fu J. C., Kass R. E. The exponential rates of convergence of posterior distributions. Ann. Inst. Statist. Math., 1988, v. 40, № 4, p. 683-691.

Поступила в редакцию

21. VII. 2000

Исправленный вариант

10.IV.2002 